\title{
Los retos educativos durante la pandemia de COVID-19: segunda encuesta a profesoras y profesores de la UNAM
}

\author{
Mercedes de Agüero Servín, Mario Alberto Benavides Lara, \\ Jesús Rendón Cazales, Maura Pompa Mansilla, Alan Kristian \\ Hernández-Romo, Ana María del Pilar Martínez-Hernández y \\ Melchor Sánchez-Mendiola
}

\section{Resumen}

Objetivo: identificar las problemáticas, necesidades de formación, herramientas digitales y prácticas de enseñanza -interacciones y evaluación- de los profesores de la UNAM durante los tres primeros meses de confinamiento por la pandemia.

Método: estudio exploratorio y descriptivo que presenta los principales resultados cuantitativos de una segunda encuesta aplicada en junio de 2020, a una muestra estratégica y por conveniencia de profesoras y profesores de la unAM ( $n=513)$ acerca de la experiencia durante el tránsito a la educación remota de emergencia. Los resultados que se presentan corresponden a los reactivos del cuestionario asociados a las problemáticas pedagógicas que enfrentaron, así como al tipo de herramientas digitales, interacciones y estrategias de evaluación que emplearon; adicionalmente se presentan los intereses y necesidades de formación que las y los profesores identificaron para transitar a una modalidad no presencial de educación.

Resultados: se encontraron diferencias de acuerdo con el nivel educativo en que enseñan los profesores, bachillerato, licenciatura o posgrado. Los profesores de bachillerato expresaron estar más confiados, se sienten más seguros y expresan estar mejor equipados.

Conclusiones: las y los profesores pudieron transitar hacia la educación remota, pero las prácticas tradicionales en el aula, a los tres primeros meses de educación remota de emergencia fueron las preferidas y las que se trasladaron como formas de interacción y evaluación a la educación digital, mostrándose con esto que la mayoría de las y los profesores carecen de elementos formativos y de profesionalización en enseñanza y aprendizaje universitarios lo suficientemente extendidas y apropiadas como para diseñar actividades de aprendizaje acordes con métodos de enseñanza y diseño de estrategias de aprendizaje más horizontales, dialógicas y constructivas; las cuales llevan décadas demostrando ser más efectivas, pertinentes y acordes a la educación profesional.

Palabras clave: educación superior, covid-19, estrategias educativas, educación a distancia, tecnología de la información de la comunicación. 


\title{
EDUCATIONAL CHALLENGES DURING THE COVID-19 PANDEMIC: SECOND SURVEY OF UNAM TEACHERS
}

\begin{abstract}
Objective: to identify, through a follow-up survey, the problems, faculty educational needs, digital tools, and teaching practices -interactions and evaluation-, of UnAm's faculty during the first three months of confinement due to the pandemic.

Method: an exploratory and descriptive study was conducted that presents the main quantitative results of a second survey applied in June 2020 to a strategic and convenience sample of UnAm teachers ( $n=513$ ), about their educational experience during the transition to emergency remote education. The results presented here correspond to the questionnaire items associated with the pedagogical problems that they dealt with, as well as the type of digital tools, interactions, and assessment strategies that they used; additionally, the interests and training needs teachers identified to transition to a non-face-to-face modality of education are presented.
\end{abstract}

Results: differences were found in relation with the educational level in which faculty teach, baccalaureate (or high school), undergraduate or graduate. High school teachers were more confident, felt securer, and better equipped.

Conclusions: Teachers were able to move towards remote education, but traditional classroom practices, the first three months of emergency remote education were preferred, also those that were transferred as forms of interaction and evaluation to digital education; most of the teachers lack prior training and professionalization for university teaching and learning, that would be sufficiently extended and appropriate to design learning activities in accordance with teaching methods, they don't design more horizontal, dialogical and constructive learning strategies; all of which have been proved to be more effective, relevant and consistent with professional education for decades.

Keywords: Higher Education, covid-19, Educational Strategies, Distance Education, Information Technology. 


\section{Mercedes de Agüero Servín}

Licenciada en Pedagogía por la Facultad de Filosofía y Letras de la Universidad Nacional Autónoma de México (UnAm, 1980-1983), con un diplomado en Educación Especial en la Universidad Iberoamericana (UIA, 1987-1988), con maestría en Investigación y Desarrollo de la Educación en la UIA (1989-1991), y con el doctorado interinstitucional en Educación de la Universidad Autónoma de Aguascalientes (1997-2001). Cuenta con un diplomado en violencia contra las mujeres por la Asociación de Desarrollo Integral para Personas Violadas y el Instituto de Desarrollo Social (ADIVAC-Indesol). Fue miembro del Sistema Nacional de Investigadores (SNI) de 2000 a 2012. Está certificada internacionalmente como psicoterapeuta corporal (2013-2015) por el Institute of Core Energetics de Nueva York, y a nivel nacional por Corpore de México, A. C. Tiene un posgrado en Psicología Transpersonal (2016-2017) por el Transformational Training Institute de Nueva York y Corpore de México, A. C. Actualmente se desempeña como la Subdirectora de Investigación en Educación, en la Coordinación de Universidad Abierta Innovación Educativa y Educación a Distancia de la unAm.

\section{Mario Alberto Benavides Lara}

Licenciado y maestro en pedagogía por la UnAM y con estudios en la maestría en política pública comparada en Flacso. Cuenta con experiencia de 15 años en los distintos niveles de la educación formal, así como en ámbitos de la educación no formal. Autor y coautor de varios artículos y capítulos de libros en educación. Tiene experiencia en el diseño e implementación de estrategias de intervención educativa, acompañamiento docente, políticas educativas, formación ciudadana, didáctica de las ciencias sociales y evaluación curricular. Está interesado en conocer y aportar en el conocimiento de los docentes y su acercamiento a la cultura científica y académica. Actualmente se desempeña como el coordinador de Investigación en Educación, en la Coordinación de Universidad Abierta Innovación Educativa y Educación a Distancia de la UnAM.

Jesús Rendón Cazales

victor_rendon@cuaieed.unam.mx orcid.org/0000-0001-7937-562X

Doctor en Ciencias con especialidad en Investigaciones Educativas por el Departamento de Investigaciones Educativas del Cinvestav del Instituto Politécnico Nacional (IPN). Especialista en temas relacionados con prácticas sociales de lectura y escritura, cultura digital, desarrollo profesional docente y vinculación entre educación formal y no formal. Ha participado en diferentes proyectos de investigación y tiene publicaciones relacionadas con la apropiación de tecnologías digitales en ámbitos educativos. Actualmente se desempeña como el coordinador de Investigación Traslacional en Educación, en la Coordinación de Universidad Abierta Innovación Educativa y Educación a Distancia de la unAM.

\section{Maura Pompa Mansilla}

maura_pompa@cuaieed.unam.mx orcid.org/0000-0002-7188-1090

Licenciada en Pedagogía por la Facultad de Filosofía y Letras de la unAM. Ha trabajado desde hace más de 14 años en instituciones del sector educativo, ocho de los cuales fue investigadora en el Centro de Estudios Educativos A. C. (CEE). 
Ha realizado investigación vinculada al ámbito educativo en temas relacionados con equidad, educación indígena, evaluación y desempeño docente, inter y multiculturalidad, identidad del profesorado e inclusión de las TIC en el aula; también se ha desempeñado en el área de evaluación educativa. Cuenta con publicaciones al respecto en libros y revistas. Ha impartido diversos cursos y talleres sobre las temáticas antes señaladas, y participado en congresos en México y el extranjero. Es docente de posgrado y actualmente se desempeña como jefa del Departamento de Investigación Traslacional en Educación, en la Coordinación de Universidad Abierta Innovación Educativa y Educación a Distancia de la unAm.

\section{Alan Kristian Hernández-Romo}

alan_hernandez@cuaieed.unam.mx orcid.org/0000-0002-4299-8628

Es Médico Veterinario Zootecnista, Maestro en Administración, con especialidad en Política y Gestión Ambiental, y Maestro en Educación en Ciencias de la Salud. Sus temas de interés son la investigación en educación, innovación educativa y evaluación educativa en las Ciencias de la Salud. Actualmente es Coordinador de Gestión y Planeación en la Coordinación de Universidad Abierta, Innovación Educativa y Educación a Distancia (CUAIEED) de la unAm.

\section{Ana María del Pilar Martínez-Hernández}

pilar_martinez@cuaieed.unam.mx orcid.org/0000-0001-6612-2252

Licenciada, maestra y doctoranda en Pedagogía por la Facultad de Filosofía y Letras de la unam. Profesora de carrera en el Colegio de Pedagogía de la misma Facultad. En la UNAM, se ha desempeñado como Secretaria Académica del Seminario de Pedagogía Universitaria de la Facultad de Filosofía y Letras (1991 a la fecha); Coordinadora del Colegio de Pedagogía de la División de Estudios Profesionales (1994 a 1998, y 2005 a 2011); Subdirectora Técnica en la Dirección General de Desarrollo Institucional de la Secretaría de Planeación (1998-2000); y Coordinadora de Planeación del Programa Universitario de Estudios de Género de la Coordinación de Humanidades (abril 2000 - marzo 2004). Asimismo, fue consejera universitaria representante del profesorado de la Facultad de Filosofía y Letras (2012-2016). Actualmente se desempeña como directora de Innovación Educativa, Desarrollo Curricular y Formación Docente en la Coordinación de Universidad Abierta Innovación Educativa y Educación a Distancia de la UNAM.

\section{Melchor Sánchez-Mendiola}

melchor_sanchez@cuaieed.unam.mx
orcid.org/0000-0002-9664-3208

Doctor en Ciencias y en Educación en Ciencias de la Salud (UNAM); maestro en Educación en Profesiones de la Salud por la Universidad de Illinois; médico militar y pediatra de la Escuela Médico Militar de la Universidad del Ejército y Fuerza Aérea. Fellow en Investigación Clínica, Hospital General de Massachusetts, Boston, y Centro de Investigación Clínica, Instituto Tecnológico de Massachusetts, Cambridge, Estados Unidos. Profesor Titular "B" Tc definitivo en la Facultad de Medicina (UNAM). Coordina la maestría y el doctorado en Educación en Ciencias de la Salud de esta facultad. Miembro de la Academia Nacional de Medicina de México; investigador nacional nivel 1, sNI. Actualmente es Coordinador de Universidad Abierta Innovación Educativa y Educación a Distancia de la unAm. 
Esta publicación se elabora y difunde como contribución a la $3^{\text {a }}$ Conferencia Mundial de Educación Superior, organizada por la UNESCO del 18 al 20 de mayo de 2022, con el fin de mejorar la contribución de las instituciones y los sistemas de educación superior de todo el mundo, en el marco de la Agenda 2030 para el Desarrollo Sostenible, su promesa de no dejar a nadie atrás, y con la mirada dirigida hacia los futuros de la educación. El contenido de esta publicación no expresa necesariamente las opiniones de la UNESCO ni de sus Estados Miembros. 


\section{Introducción}

En marzo del año 2020 la entonces Coordinación de Desarrollo Educativo e Innovación Curricular (CODEIC) de la Universidad Nacional Autónoma de México (UNAM) lanzó la invitación a una muestra de profesoras y profesores de los niveles de bachillerato y licenciatura para contestar un cuestionario, con el fin de identificar los retos de índole pedagógico, logístico y tecnológico a los que en el tránsito a la educación remota de emergencia las y los profesores de la unAM se estaban enfrentando (Sánchez-Mendiola et al., 2020).

En junio del mismo año se creó por acuerdo del Rector de la unAm la Coordinación de Universidad Abierta, Innovación Educativa y Educación a Distancia (CUAIEED) que surgió a partir de una reorganización administrativa y estratégica de la propia universidad. Se integró a la entonces Coordinación de Universidad Abierta y Educación a Distancia (CUAED) y a la Coordinación de Desarrollo Educativo e Innovación Curricular (CODEIc) con el propósito de, entre otras cosas, atender de manera más eficiente las situaciones que la propia pandemia había generado en el ámbito educativo e institucional de la Universidad (Gaceta unAm, 2020). Con esto, lo que se buscó fue avanzar y acortar la distancia en torno a los modelos que históricamente habían separado a los subsistemas en los que se ofertan los programas formativos de la unAm especialmente en la licenciatura (Amador, 2012).

Uno de los primeros proyectos al que se le dio continuidad en la nueva dependencia fue mantener el trabajo de indagación y análisis que la contingencia sanitaria estaba registrando en las prácticas educativas de la universidad. Así, se decidió dar continuidad a un primer ejercicio (Sánchez-Mendiola et al., 2020) y construir un segundo cuestionario que pusiera especial énfasis en los aspectos de carácter didáctico-pedagógicos (Cáceres, et al., 2003; Contreras, 1994; Díaz Barriga, 2005) que la contingencia, la educación remota de emergencia y las prácticas educativas - muchas de ellas basadas en un planteamiento tradicional del ejercicio docente- estaban registrando.

En junio de 2020 se liberó un segundo cuestionario más extenso, que rescatara en profundidad los aspectos que se exploraron en el primer cuestionario de marzo del mismo año, al inicio del confinamiento. Entre las principales conclusiones de este primer estudio está el reconocer la importancia de garantizar el acceso a internet y la cultura digital, como una condición necesaria para que las y los profesoresy sus estudiantes incorporen las tecnologías digitales en sus prácticas educativas (Reich, 2020; Sánchez Mendiola, et al., 2020).

Con la intención de caracterizar de manera más completa estas prácticas de las y los profesores, este segundo cuestionario se diseñó con base en una matriz de las dimensiones y constructos que se abordan. Entre otros asuntos se decidió incluir y definir las problemáticas educativas y personales a las que se han enfrentado las y los docentes, el tipo de herramientas empleadas, las interacciones didácticas desarrolladas y las estrategias de evaluación empleadas. 
A más de un año de declarada formalmente la clausura de las actividades sociales de casi todo el mundo y ante la posibilidad de regresar a las instalaciones universitarias, parece que la Universidad se encuentra ante la posibilidad de intentar retornar a una situación didáctico-pedagógica similar a la que existía previa a la contingencia. Normalidad caracterizada por las inercias y las formas de enseñanza tradicionales a las que están atadas las instituciones educativas, incluida la universidad y el modelo en el cual se basa, o bien, transitar hacia una educación en donde lo digital, lo multi e intermodal y la posibilidad de generar prácticas menos prescriptivas y tradicionales en la relación docente - estudiante hacia otras que sean un horizonte al cual se aspira (Cobo et al., 2020; Díaz Barriga, 2020; Grijalva-Verdugo, A. y Lara-Rivera, J., 2021; Kress et al., 2009; Tesar, 2020).

Para imaginar, diseñar y arribar a una nueva realidad post confinamiento por la pandemia, con una estrategia funcional a las necesidades de aprendizaje y enseñanza, no bastará con que la universidad o sus recursos se dirijan a la adopción ciega y acrítica de un nuevo modelo educativo o modificaciones a los actuales modelos - escolarizado, abierto y a distancia - que suponga que la tecnología en educación genera por sí sola nuevas prácticas pedagógicas. Por el contrario, transitar a modalidades o el diseño de un modelo de educación basados en lo digital implica reconocer que hay otros factores que contribuyen a que las y los estudiantes tengan una experiencia de aprendizaje efectiva, relevante, pertinente y satisfactoria (Gopal et al., Sieber, 2005; 2021; VelázquezCigarroa, E. y Tello-García, E., 2021). El cambio que la universidad requiere para atender la docencia de acuerdo con los retos y desafíos que plantea la pandemia apela a una reforma profunda de las bases didácticas y pedagógicas en las que se ha sostenido el quehacer universitario y que se complejiza con las características particulares de la universidad y de la diversidad de entidades académicas que la conforman (Clark, 1997). La unam es una institución de educación superior (IES) que está atravesada por aspectos organizacionales, así como políticos, etarios y de género por mencionar sólo algunos aspectos que condicionan el tipo de respuestas que la universidad puede desplegar, pero que es necesario considerar a fin de construir propuestas lo más pertinentes y efectivas posibles para la continuidad de los procesos de enseñanza y aprendizaje, así como la diversidad de prácticas educativas de la Universidad.

Aunque en un inicio se adoptó a la educación remota de emergencia como el marco analítico desde el cual se diseñaron y justificaron las múltiples iniciativas que las instituciones de educación han desarrollado y las innovaciones educativas que se diseñaron en estos meses (Hodges, et al., 2020; Mae-Toquero, 2020; Trust, et al., 2020 y Whittle et al., 2020). También, surgió una corriente crítica que se cuestionaba hasta dónde la emergencia entendida como un accidente o contingencia se podía extender y hasta donde esta emergencia había terminado por volverse una constante y nueva "normalidad", así como una condición de los sistemas educativos que afectó en especial a las personas con mayores desventajas sociales y tecnológicas (Aguilera et al., 2020; Luke et al., 2021; Pardo et al., 2020). Al punto de que la pandemia no ha hecho más que desnudar las carencias que la educación escolarizada venía arrastrando e 
invisibilizando de distintas formas aún no suficientemente estudiadas, pero que la normalidad a la que todas y todos nos habíamos acostumbrado porque nos hemos desarrollado en ella, en realidad estaba enmascarando (Alasuutari, 2020; CLADE / IIPE-UNESCO / OXFAM, 2021).

Bajo este contexto el principal reto parece estar en el desafío de no regresar a una normalidad previa a la pandemia con todos su vicios e inercias, sino que estos 15 meses sean un catalizador de cambios profundos en la concepción y prácticas de enseñanza y aprendizaje que se movieron en la sociedad durante la pandemia a partir de la evidencia que hasta el momento se ha acumulado (CEPAL-UneSCO, 2020; OECD, 2021; Pokhrel et al., 2021). El cambio en el hacer de las universidades puede obedecer, en tanto institución, a un cambio incremental (Lindblom, 1958) o bien a partir de shocks internosy externos (Sabatier, 1991) que llevan a las Ies y a la universidad a una situación de crisis en la que la transformación sea la vía para consolidar las nuevas prácticas digitales y epistemologías de la educación de docentes y estudiantes. La pandemia no debe leerse como una singularidad que no volverá a ocurrir, la vacuna no es la panacea para el regreso sino la puerta a una nueva forma de organizar las actividades sociales y en esa medida modificar las relaciones políticas, económicas, sociales y educativas que tienen lugar a niveles micro, meso y macrosociales (Moore, 2021; PNUD, 2020).

Parece queen el centro de la discusión del regreso a la Universidad seencuentra la necesidad de una reforma pedagógica que necesita involucrar e impactar al resto de las estructuras organizativas de la sociedad, para ello se requiere capitalizar lo que en esta coyuntura se ha avanzado en la digitalización de la educación (Loreto, 2021; Saavedra, 2021; Silas et al., 2020). Alcanzar con calidad educativa los retos y demandas, en el caso de la unAM, implica generar la información necesaria para actores y estructuras institucionales, también de interés pedagógico acerca de lo que está ocurriendo que aporte a un modelo de toma de decisiones basado en la evidencia (Camilli-Trujillo, et al., 2020; Davies et al., 1999; Flores, 2013). El estudio cuyos resultados aquí se presentan se suman a los publicados de manera anterior al inicio de la pandemia con la intención de aportar información que permita orientar no sólo el regreso a lo presencial sino a los cambios que en el corto y mediano plazos habrá de registrar la Universidad y las IES.

En el presente artículo se rescatan los principales hallazgos relacionados con el tipo de prácticas que las y los docentes de la unam declararon registrar a tres meses (marzo a junio) de iniciada la contingencia sanitaria por covid-19. Con este propósito se construyó una pregunta que orientó el análisis de los resultados:

¿Qué tipo de problemáticas, necesidades de formación, herramientas digitales y prácticas de enseñanza (interacciones y evaluación) han registrado las y los profesores de la UNAM durante el primer semestre en el que transitaron hacia un modelo de educación remota no presencial?

En la medida de lo posible se ha tratado de incluir, en los casos que así lo ameritan, las diferencias en las respuestas que ofrecieron los profesores a partir 
del nivel educativo al que pertenecen (bachillerato, licenciatura y posgrado). El estudio busca reconocer las características institucionales, organizativas y didácticas-pedagógicas que orientan el trabajo en cada nivel y lo dotan tanto de su especificidad como de sus retos.

\section{Método}

\section{Tipo de estudio}

El presente estudio se define como de tipo exploratorio, descriptivo y de corte mixto, ya que incorpora reactivos que se analizaron bajo un enfoque estadístico descriptivo como reactivos abiertos que se analizaron bajo un método de análisis crítico del discurso. se presentan los resultados de los reactivos cerrados y de opción múltiple de respuesta, por lo que las preguntas abiertas de análisis cualitativo no son abordadas en este texto.

\section{Muestreo}

La muestra se diseñó, de acuerdo con Etikan et al. (2016), por criterios de conveniencia y estratégicos. Respecto al tamaño de la muestra " $\mathrm{n}$ " esta corresponde a 513 respuestas de profesoras y profesores de educación media superior o bachillerato y de licenciatura, tanto de tiempo completo como de asignatura (contratados por horas).

\section{Estrategia de levantamiento de datos}

Dado que la UNAM presenta una estructura organizativa y administrativa muy compleja que en ocasiones requiere la coordinación de muchas dependencias de la universidad, y ante el reto de generar información de manera expedita en una coyuntura marcada por un fenómeno social inédito, se decidió diseñar una estrategia de levantamiento de datos basada en los recursos que la propia dependencia tenía disponibles. De esta manera se elaboró una estrategia basada en el envío de invitaciones para contestar el cuestionario el cual se montó en la plataforma Surveymonkey.com, la base de datos de los posibles informantes se tomó de los registros que la Coordinación poseía de las y los profesores que han tomado cursos de actualización y formación docente.

\section{Instrumento}

Para captar la información de las y los profesores se diseñó un cuestionario de respuestas cerradas y abiertas dirigido a docentes que se encontraban con grupos activos al iniciar la contingencia sanitaria. El instrumento se compuso de 40 reactivos que se elaboraron como preguntas dicotómicas, baterías tipo Likert con escalas de frecuencia y acuerdo, así como preguntas abiertas.

Analíticamente el instrumento contempló cinco dimensiones de exploración de las opiniones, experiencias y experiencias de las y los profesores universitarios, mismas que se muestran en la figura 1. 
Figura 1. Dimensiones del cuestionario.

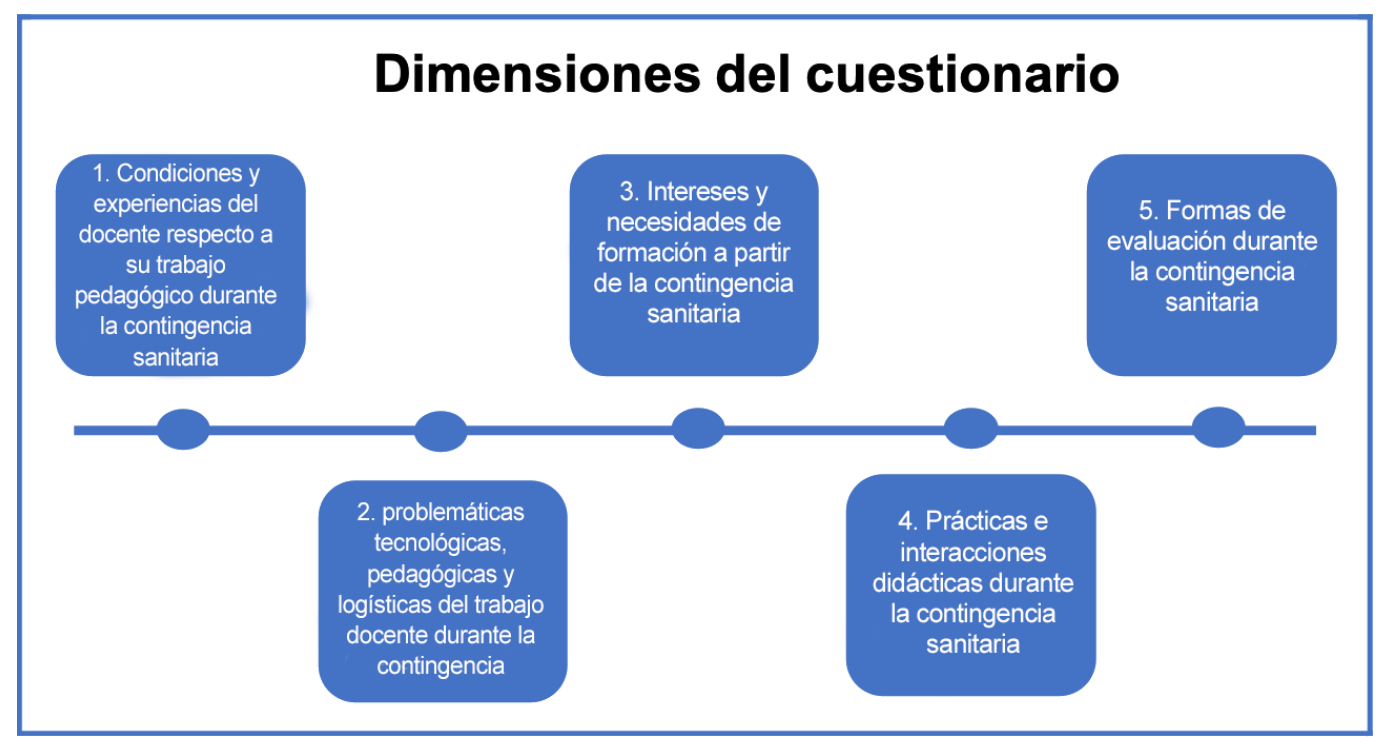

\section{Resultados}

A continuación se muestran los principales resultados cuantitativos asociados a las dimensiones 2, 3, 4 y 5. Previo a ello se incluye una caracterización del perfil de los y las informantes que participaron del llenado del cuestionario.

\section{Perfil de las y los informantes}

Del total de quienes participaron en el Ilenado del cuestionario ( $n=513$ ) 64\% correspondió a mujeres y el 36\% a hombres. Acerca de la edad 28\% se ubicó en el rango de 44 a 53 años, 27\% en el rango de 54 a 63 años, 26\% en el rango de 34 a 43 años, 13\% en el rango de 64 a 80 años y 7\% en el rango 24 a 33 años.

En cuanto al tipo de contratación 56\% fueron profesoras(es) de asignatura, $28 \%$ profesoras(es) de tiempo completo, 5\% técnicas(os) académicos y $11 \%$ declaró tener otro tipo de contratación (profesora de asignatura de medio tiempo, investigadora de medio tiempo o tiempo completo, etc.). Acerca del nivel en el que imparten clase 65\% lo hace en el nivel de licenciatura, 18\% posgrado y $14 \%$ en bachillerato. Respecto a la modalidad en la que imparten clase $85 \%$ lo hace en el subsistema y modalidad de educación presencial y escolarizada, mientras que 10\% lo hace en el subsistema de universidad abierta y 5\% en el subsistema de educación a distancia.

Acerca del perfil académico de las y los informantes, 48\% tiene estudios de maestría, 28\% de doctorado y 18\% de licenciatura. Acerca del área de conocimiento en el que imparten clase 44\% lo hace en el área de ciencias biológicas y de la salud, 23\% en humanidades y artes, 17\% ciencias sociales y 16\% en el área de ciencias fisicomatemáticas e ingenierías (figura 2) 


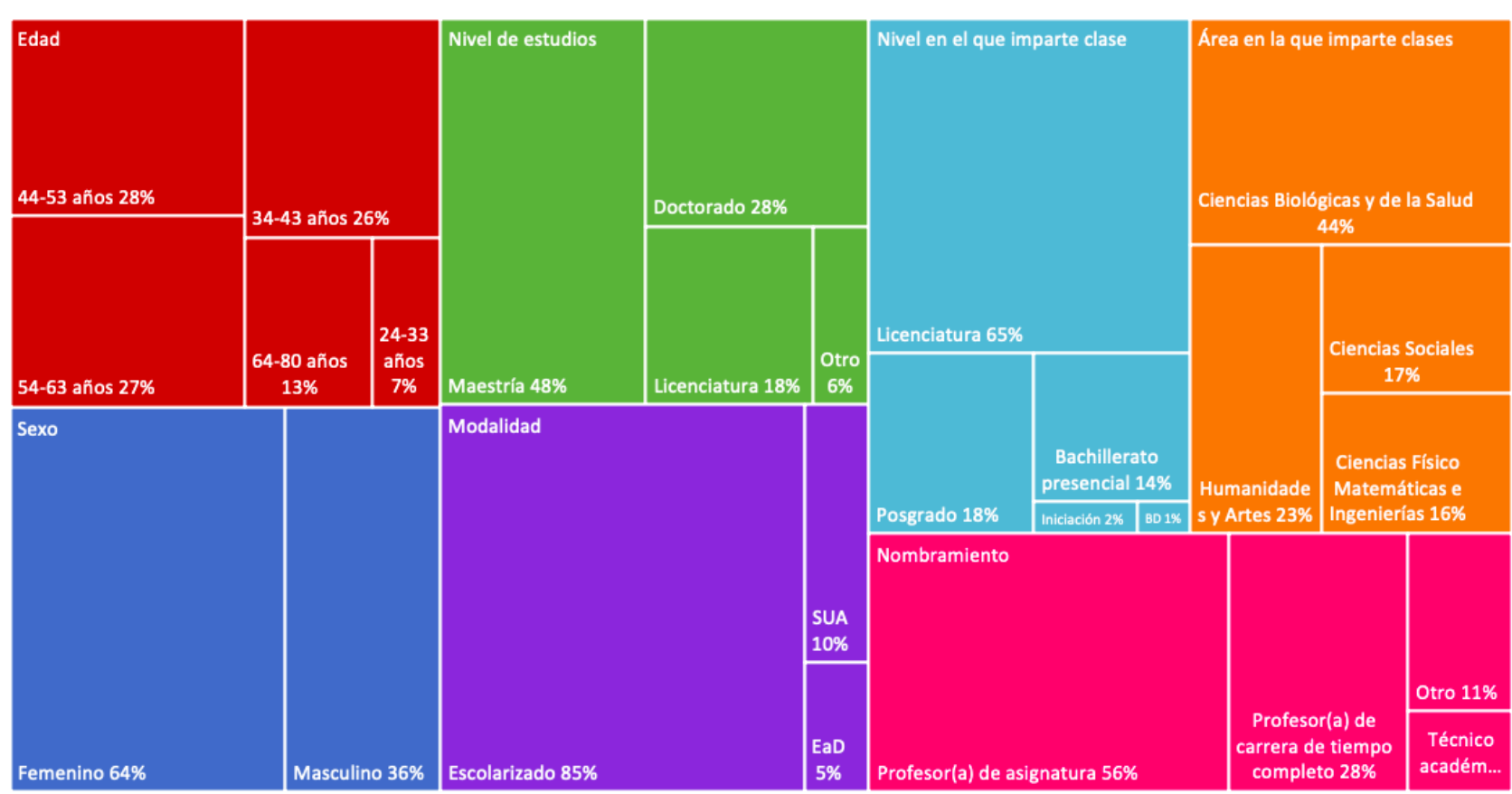

Figura 2. Perfil de las y los profesores que participaron en el estudio.
Figura 3. Problemáticas tecnológicas, pedagógicas y logísticas: equipo y conexión.

\section{Resultados cuantitativos}

\section{Problemáticas tecnológicas, pedagógicas y logísticas del trabajo docente}

En cuanto a las problemáticas a las que las profesoras y profesores se han enfrentado si bien se observa que por nivel los porcentajes son muy similares, se destaca el alto porcentaje de docentes que mencionan que su equipo de cómputo y conexión a Internet se adaptan a sus demandas de trabajo.

De esto, se destaca en la figura 3 que son las y los profesores de licenciatura los que pueden estar registrando una mayor complicación con la existencia y disponibilidad de los insumos que requieren para trabajar bajo una modalidad a distancia, mientras que en el caso del bachillerato y el posgrado entre el $85 \%$ y $87 \%$ señalaron que casi siempre o siempre su equipo de cómputo y conexión se adaptó a sus demandas de trabajo, en el caso de licenciatura sólo el 76\% declaró que esto ocurría casi siempre o siempre.

Mi equipo de cómputo y conexión a internet se adaptan a mis demandas de trabajo

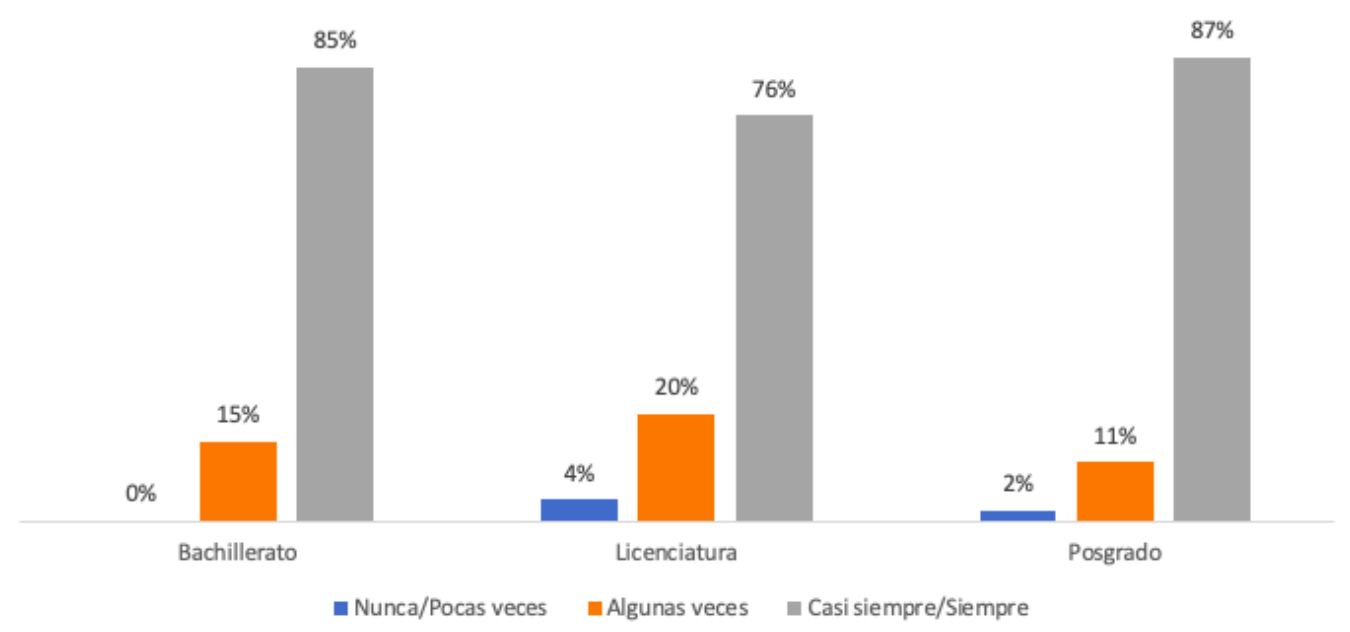


Figura 4. Problemáticas tecnológicas, pedagógicas y logísticas: conocimientos y habilidades.
Acerca del conocimiento que las y los profesores dijeron tener en el uso de herramientas y plataformas digitales para desarrollar su práctica docente no presencial se destaca el relativo bajo porcentaje (56\%) de docentes del nivel de bachillerato que dijo que esto casi siempre o siempre era así (figura 4); en comparación con el $72 \%$ de licenciatura y $71 \%$ de posgrado dijo que esto ocurría casi siempre o siempre. Un estudio posterior necesita corroborar esta diferencia porcentual de 15 puntos entre los tres niveles, puesto que la muestra de profesores de bachillerato y la de posgrado son pequeñas con relación a los de licenciatura.

El conocimiento y habilidades que poseo en el uso de herramientas y plataformas digitales me permite desarrollar mi práctica docente de manera no presencial

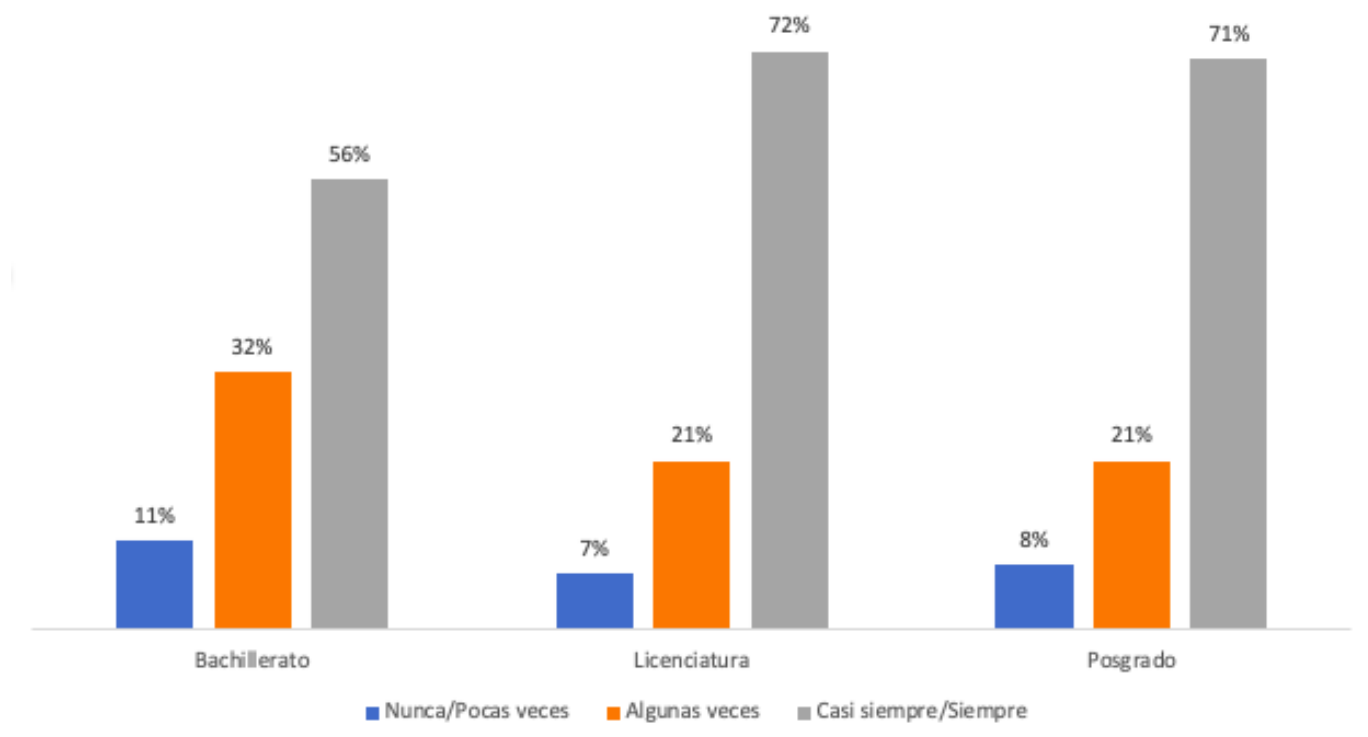

De las distintas problemáticas que se les presentaron a las y los docentes se observa (figura 5) que los menores porcentajes respecto a otras problemáticas que se ubican en el rango de casi siempre o siempre, son las relativas a la pertinencia didáctica de las actividades que las profesoras y profesores implementaron a tres meses de iniciada la pandemia.

Como se observa en la figura 5, cuando se les preguntó si consideraban que las actividades que las y los profesores desarrollaron permiten a las y los estudiantes lograr los aprendizajes de sus cursos bajo una modalidad no presencial, el 69\% de las y los profesores de posgrado, el 67\% de profesoras y profesores de licenciatura y el $65 \%$ de las profesoras y los profesores de bachillerato dijeron que esto casi siempre o siempre era así. 
Figura 5. Problemáticas tecnológicas, pedagógicas y logísticas: logro aprendizajes.
Figura 6. Intereses y necesidades de formación.
Las actividades permiten a los estudiantes lograr los aprendizajes de mi curso en modalidad no presencial

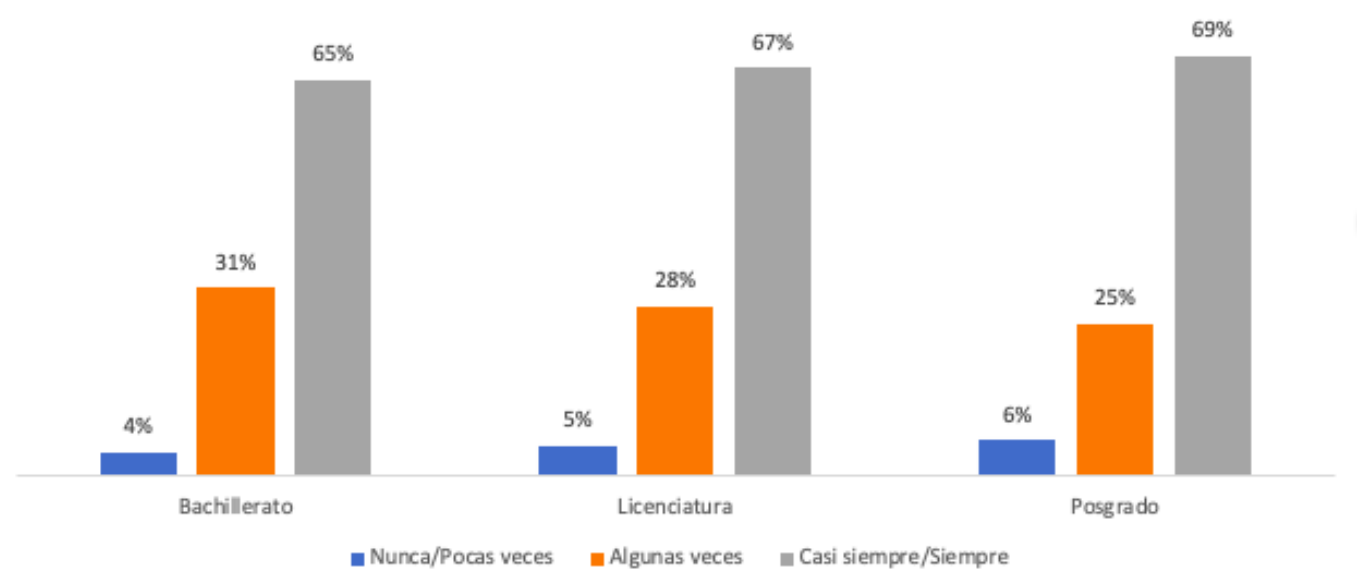

\section{Intereses y necesidades de formación}

Acerca de los intereses y necesidades de formación que las y los profesores señalaron tener en el contexto de la contingencia sanitaria se destaca que en el caso de bachillerato la temática en la que presentan mayor interés es en el "diseño de situaciones didácticas para educación no presencial" con 18\%, esta misma situación es la que también presenta el mayor interés en licenciatura con 19\% junto con la temática de "instrumentos y estrategias de evaluación para la educación no presencial". Mientras que en posgrado la temática de mayor interés es el "uso educativo del aula virtual" con 20\% (figura 6).

En contraste, la temática que registró menor interés por parte de las profesoras y profesores fue la "incorporación de la perspectiva de género en la enseñanza", ya que sólo 10\% de docentes de bachillerato dijo estar interesado, porcentaje que se reduce a $7 \%$ en el caso de posgrado y $6 \%$ en licenciatura.

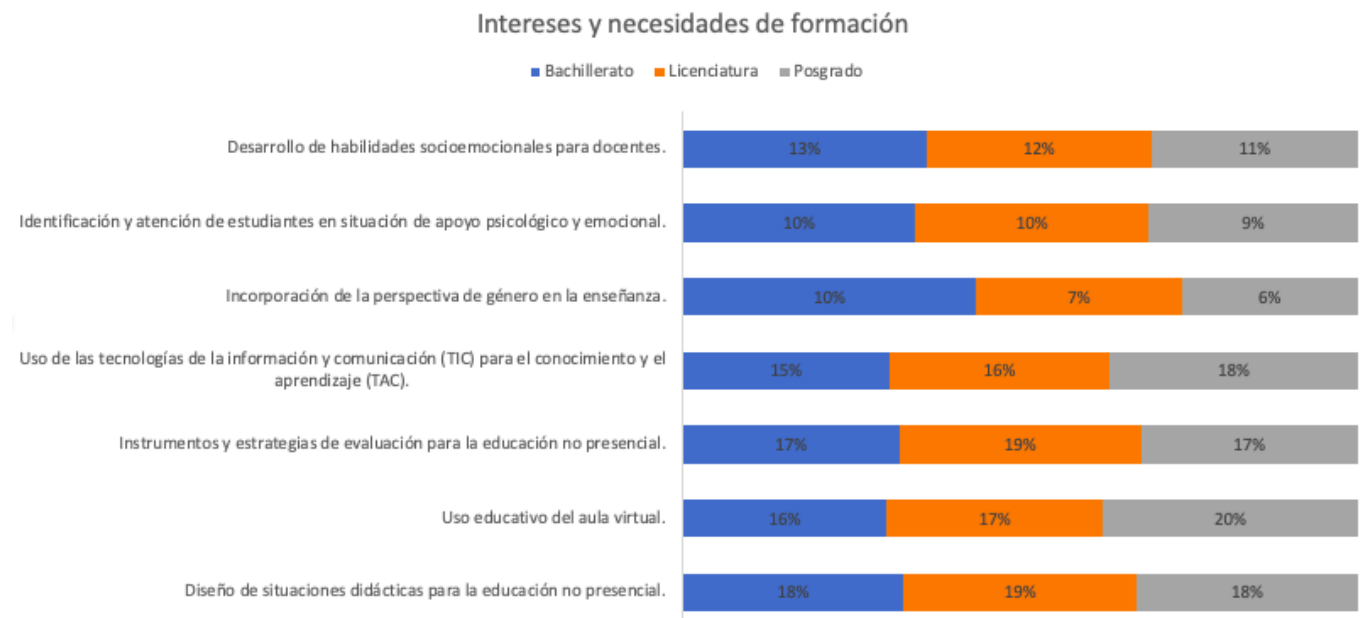




\section{Herramientas, prácticas e interacciones didácticas}

Se incluye en este apartado el tipo de herramientas didácticas que las y los profesores señalaron utilizar con mayor frecuencia en sus clases no presenciales, ya que se considera que esta información puede ayudar a comprender el tipo de prácticas e interacciones que desarrollan en sus clases.

De esta manera se observa la diferencia entre el uso de libros digitales como las herramientas que se usan con mayor frecuencia en posgrado con 57\% y licenciatura con 49\%; mientras que en bachillerato la herramienta digital más empleada son los videos con 53\%. Resalta que, ante las diferencias de las herramientas señaladas, las páginas web generales presentan prácticamente la misma frecuencia de uso en los tres niveles (figura 7).

Herramientas digitales

Figura 7. Herramientas digitales.

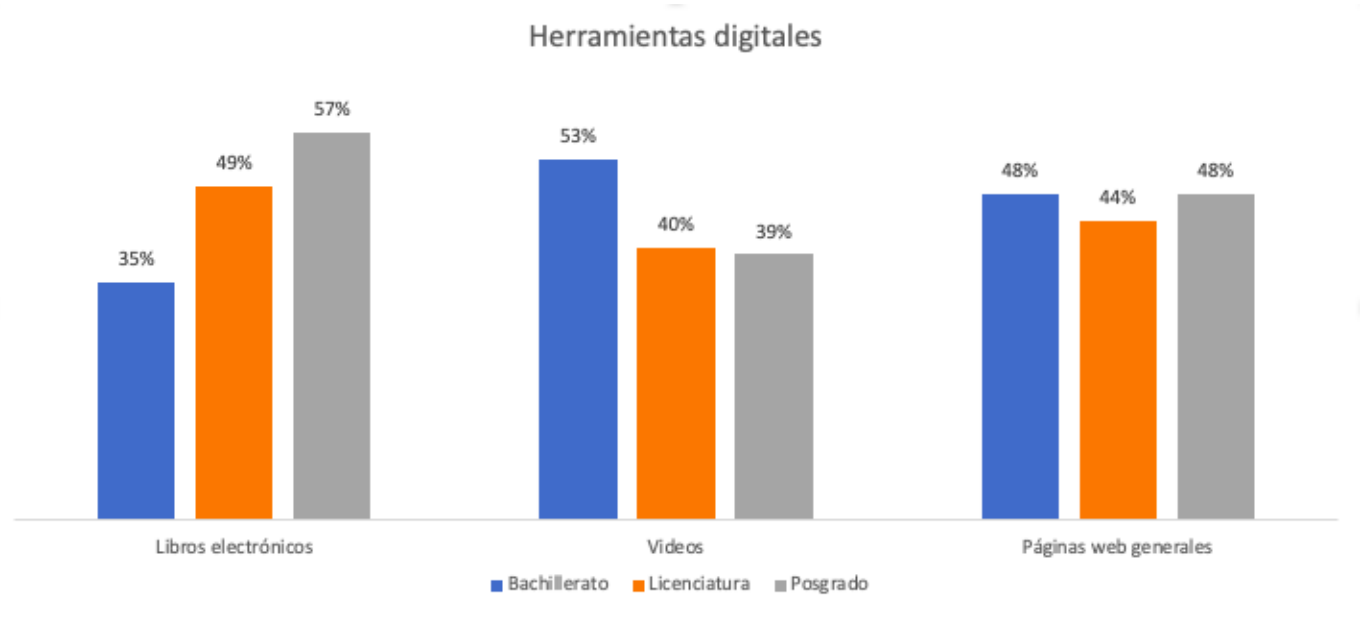

Con relación a las prácticas e interacciones que las y los profesores indicaron registrar durante la contingencia se destaca la preeminencia de la clase grupal en los niveles de posgrado con $74 \%$ entre quienes la emplean casi siempre y siempre, seguido del nivel licenciatura con $64 \%$ y del bachillerato con un $24 \%$ que indicó que la empleaban casi siempre y siempre (figura 8).

\section{Clase grupal}

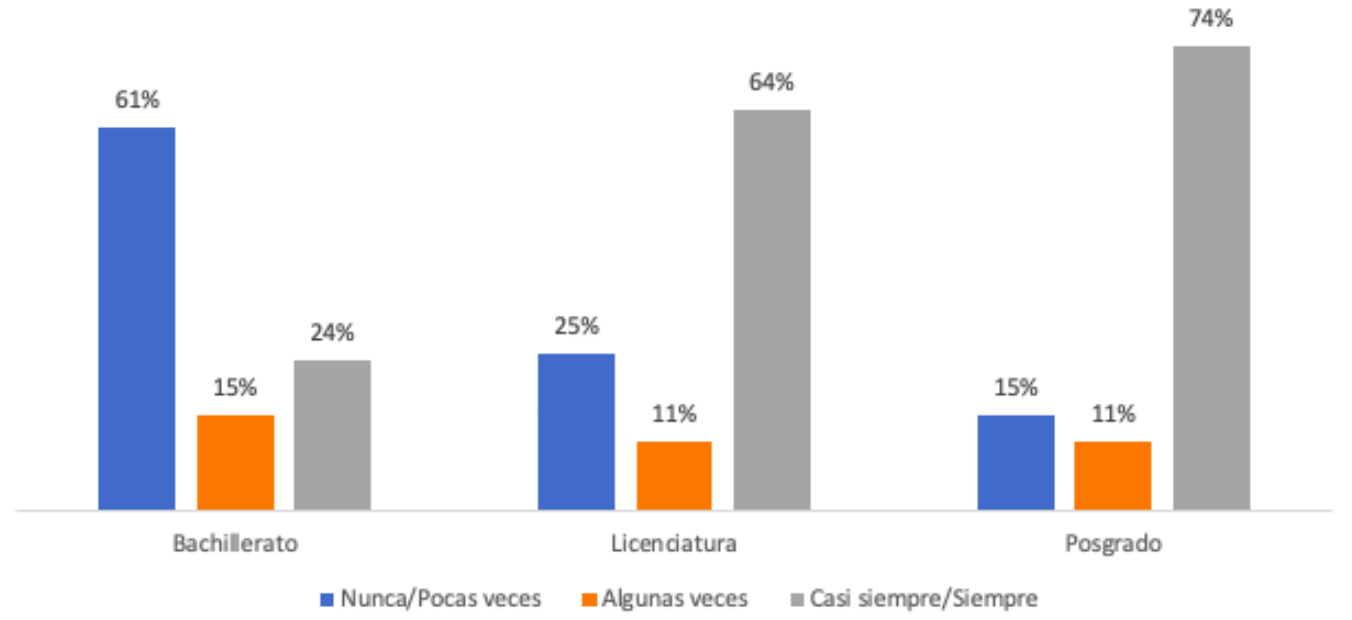


Figura 9. Prácticas e interacciones: tareas individuales.

Figura 10. Prácticas e interacciones: trabajo colaborativo.

\section{Tareas individuales}

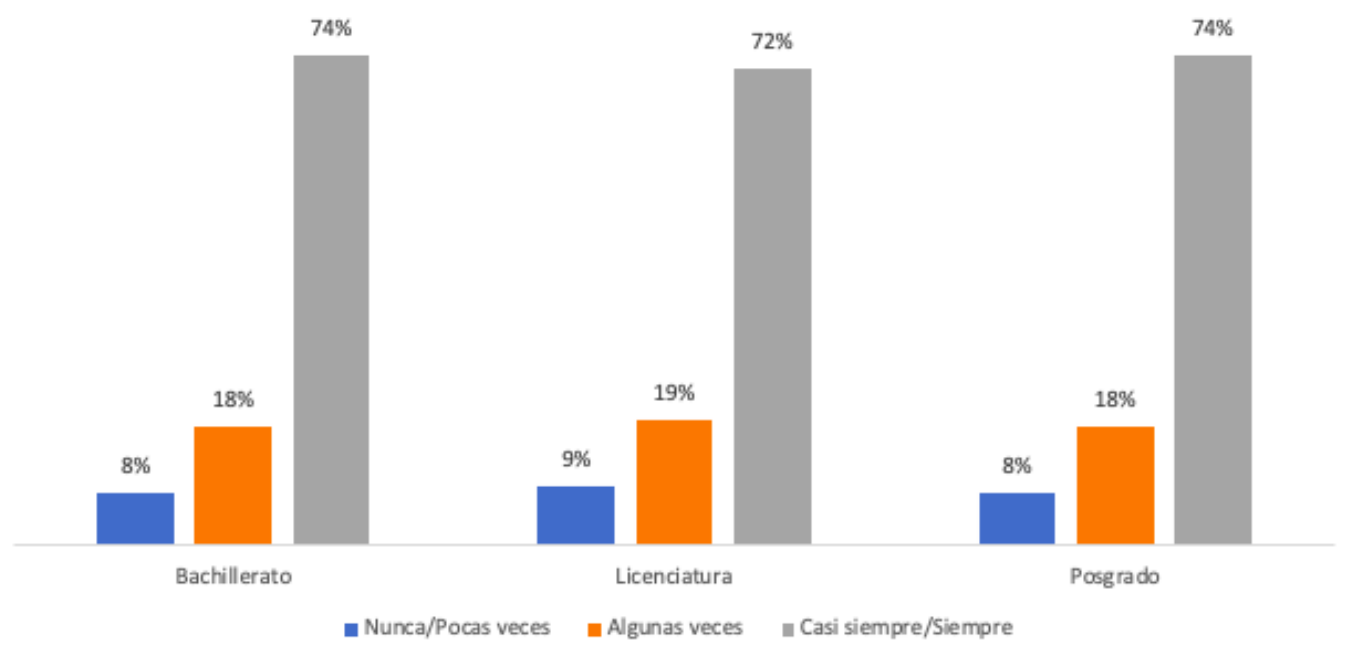

En cuanto a la frecuencia con la que las y los docentes señalaron que emplean el trabajo colaborativo durante su clase, destaca que no es muy frecuente en ninguno de los tres niveles. Mientras que en licenciatura y posgrado 36\% señaló que lo empleó casi siempre y siempre, en bachillerato 33\% señaló que lo empleó con la misma frecuencia. Sin embargo, contrasta que 54\% en nivel bachillerato dijo haberlo empleado nunca y pocas veces; y el $45 \%$ de posgrado y licenciatura que no recurre a prácticas e interacciones colaborativas (figura 10).

Trabajo colaborativo entre estudiantes durante la clase

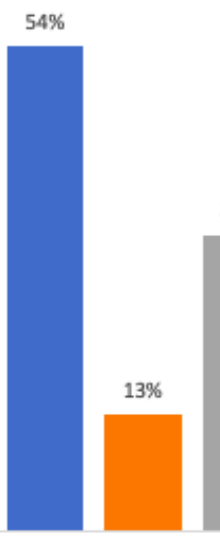

Bachillerato
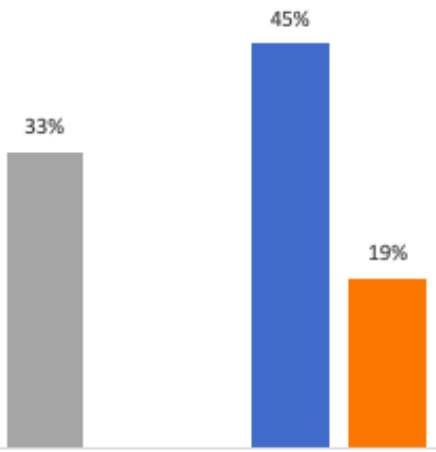

Licenciatura

- Nunca/Pocas veces

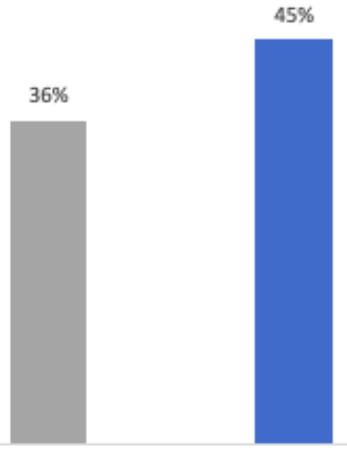

$45 \%$

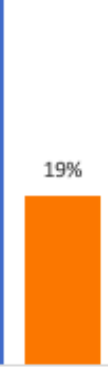

Posgrado

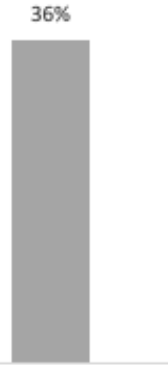

- Casi siempre/Siempre 


\section{Estrategias de evaluación}

Con relación a las estrategias de evaluación que las y los profesores señalaron emplear se observa que sí difieren por el nivel educativo del que se trate. Mientras que la investigación es la estrategia empleada con mayor frecuencia en licenciatura con 53\% y posgrado con 64\%, en bachillerato la estrategia más utilizada es la rúbrica con 57\%. Cabe resaltar que la resolución de problemas es la estrategia de evaluación que se comparte en los tres niveles educativos con porcentajes similares.

Llama la atención que en todos los niveles el empleo de estrategias como la autoevaluación y la coevaluación se usan poco, así el 32\% de los profesores de bachillerato dice que casi siempre y siempre usa la autoevaluación, el 27\% en posgrado y solamente un $23 \%$ en licenciatura. De igual manera son los docentes del bachillerato quienes emplean más la coevaluación, aunque el porcentaje no es demasiado alto pues sólo 26\% lo usa casi siempre y siempre (figura 11).

Estrategias de evaluación

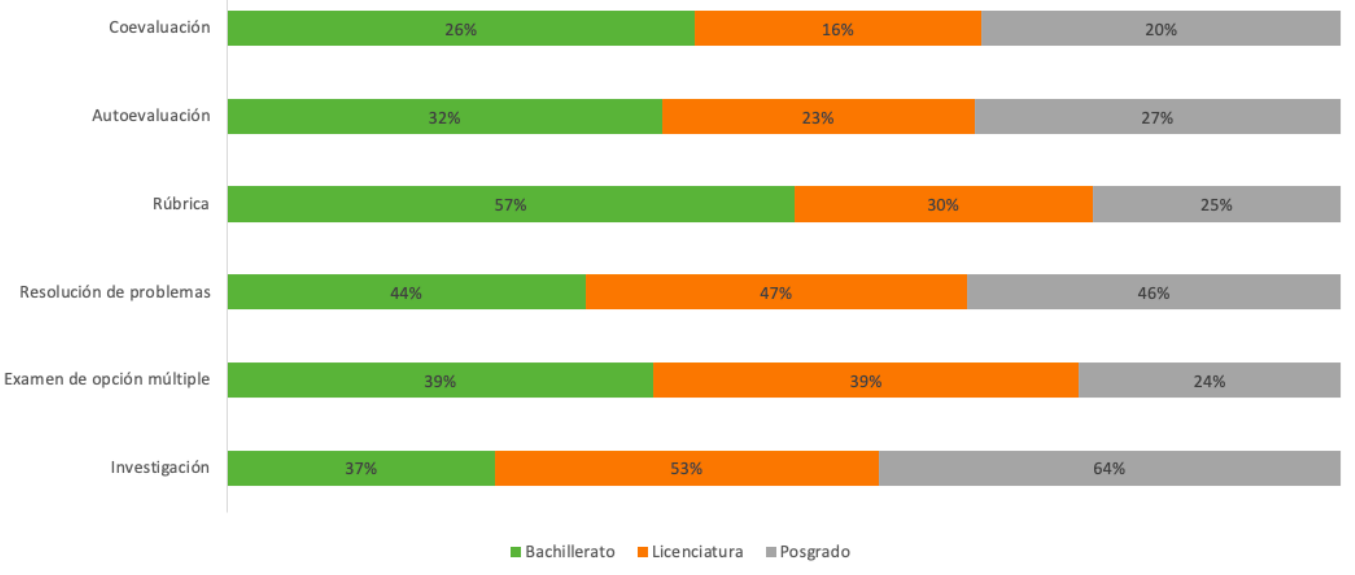

\section{Discusión y conclusiones}

Los resultados del cuestionario indican que, si bien los docentes pudieron salir adelante con su trabajo de enseñanza, la significatividad para el aprendizaje de sus estudiantes pudo estar restringida. A propósito de esto, es importante que los resultados mostrados en la figura 3 sean leídos a la luz de los otros resultados y con cautela respecto a las diferencias muestrales por nivel educativo.

Cuando se observa el tipo de herramientas digitales que emplean (figura 7), la interacción didáctica que mantienen (figuras 8, 9 y 10) y el tipo de evaluación que realizan (figura 11), se evidencian las limitaciones que existen para plantear estrategias didácticas que involucren al estudiante y lo coloquen en una dinámica de mayor actividad, así como establecer una comunicación más fluida, formativa y basada en la retroalimentación, que impliquen pedagogías constructivistas y 
de profesionalización en habilidades y actitudes para el trabajo en equipo y la colaboración.

Esta aseveración se sostiene cuando se observa que la herramienta más empleada en licenciatura y posgrado es el libro electrónico, mientras que en bachillerato es el video. Herramientas que por sí mismas presentan pocas oportunidades de interacción y dependen, en gran parte, de la manera como son empleadas dentro de actividades didácticas. Al ser las tareas individuales las de mayor preferencia entre los docentes universitarios puede inferirse que los videos y los libros electrónicos se usan para el trabajo individual sea del tipo que sea. De tal modo, que las prácticas tradicionales en el aula, a los tres primeros meses de educación remota de emergencia, fueron las preferidas y las que se trasladaron como formas de interacción y evaluación a la educación digital, mostrándose con esto que la mayoría de las y los profesores carecen de elementos formativos y de profesionalización en enseñanza y aprendizaje universitarios lo suficientemente extendidas y apropiadas como para diseñar actividades de aprendizaje acordes a métodos de enseñanza y diseño de estrategias de aprendizaje más horizontales, dialógicas y constructivas; las cuales llevan décadas demostrando ser más efectivas, pertinentes y acordes a la educación profesional.

En consonancia con lo anteriory al cruzar la información de las herramientas con el tipo de interacción que las y los profesores tienen, se puede ver que es la clase grupal la predilecta, aunque con pocas posibilidades de desarrollar trabajo colaborativo como se puede observar en la figura 11 y que constata en el uso de las tareas individuales como el tipo de práctica empleado con mayor frecuencia en las clases (figura 10). Estos resultados coinciden con los identificados por Silas (2020) acerca de las complicaciones que han tenido las y los profesores universitarios para transitar a un modelo distinto de lo presencial, así como por lo apuntado por Reich (2020), quien señala que la tecnología por sí sola no genera cambios disruptivos en la educación y la escuela, acostumbrada más a los cambios lentos y graduales, en contraste con las pretensiones de administradores y directores quienes tratan de influir desde afuera de la escuela para generar cambios pedagógicos y de resultados de aprendizaje rápidos.

También se observa que las prácticas de evaluación siguen la misma línea, al menos en licenciatura y posgrado pues la estrategia más empleada es la investigación la cual, con sustento en los datos que arroja este cuestionario se puede suponer, se desarrolla como un proceso más individual que colectivo, manera contraria a como se realiza la investigación en contextos universitarios o profesionales. Si bien en el cuestionario no se preguntó, esto queda como una hipótesis a explorar en trabajos de corte cualitativo. ¿Es la investigación una forma de estrategia didáctica en las IEs de búsqueda de información para el aprendizaje, en contraste con una forma de formación en las competencias y actitudes de un quehacer científico? ¿Cómo conciben y usan "la investigación" los profesores en el aula, la educación a distancia y abierta del bachillerato y licenciaturas de la unAm? 
Con relación a las estrategias de evaluación empleadas se destaca el uso de la rúbrica con mucha presencia en el bachillerato, aunque no se puede determinar con exactitud el tipo de actividades en el que se les emplea. Es de destacar que, a partir de estos resultados, el nivel de bachillerato es el que registra una mayor preocupación para involucrar al estudiantado en el proceso de aprendizaje para la autorregulación y metacognición, ya que es este mismo nivel el que presenta un mayor uso de la autoevaluación y la coevaluación, aunque en todos los casos el uso de estas estrategias es poco empleado. En la licenciatura y el posgrado son la investigación y la resolución de problemas las estrategias de evaluación con más alta respuesta porcentual. En el bachillerato se utilizan mayormente la rúbrica y la solución de problemas.

Al preguntarles cuáles consideran que serían las temáticas de una oferta de formación de su interés, la mayoría de los docentes apuntó la necesidad de desarrollar capacidades pedagógicas para ejercer su práctica mediada por las tecnologías. Situación que por sí misma representa un avance en la idea acerca de que la mejor forma de que las y los profesores se apropien de las tecnologías, era capacitándose en su uso sin vincularlas ni situarlas en la práctica docente. Avanzar en la formación de las y los profesores que impacte en su motivación es crítica, pues como lo apunta Luke et al. (2021) profesores que se encuentran motivados han visto a la pandemia como un reto que ha revigorizado su práctica, comparado con aquellos que tienen poca motivación y ven a la pandemia como una amenaza a su trabajo. ¿Qué tanto la necesidad de formarse en TIC de manera aislada del contenido disciplinar o profesional es una manera de perpetuar la desvinculación epistemológica de las didácticas disciplinares universitarias?

Esta interpretación se refuerza al observar la figura 2, especialmente en el caso de las y los profesores de bachillerato, quienes mencionan que su conocimiento en el uso de herramientas y plataformas digitales no siempre les permiten desarrollar su práctica docente, respuesta que se puede considerar como reflejo de una actitud autocrítica acerca de sí mismos como docentes, que contrasta con los resultados de las y los profesores de licenciatura y posgrado. Si estos resultados son evidencia de una conciencia epistemológica diferenciada por niveles educativos, entonces: ¿cómo comprender las diferencias a favor del bachillerato?

Por otro lado, ante la situación que viven tanto la Universidad, las IEs y el país con relación a la realidad desigual y violenta a partir de las diferencias y las prácticas sociales y de educación marcadas por pautas anacrónicas de género, llama la atención y desconcierta que las y los profesores universitarios expresan poco interés por conocer e incorporar las perspectivas de género en el trabajo docente (figura 6) y apuntan en sentido contrario a lo que las agendas sociales recomiendan y acordaron que se plasma en documentos de relevancia mundial como la Convención sobre la eliminación de todas las formas de discriminación contra las mujeres (por sus siglas en inglés, la cEDAw), la Convención Interamericana para prevenir, sancionar y erradicar la violencia contra las mujeres de Belém Do 
Pará y, los principios de Yogyakarta que son los principios sobre la legislación internacional de derechos humanos en relación con la orientación sexual y la identidad de género; así como el informe de desarrollo humano del PNUD (2020).

\section{Referencias}

* Aguilera, E. y Nightengale-Lee, B. (2020). Emergency remote teaching across urban and rural contexts: perspectives on educational equity. Information and Learning Sciences, 2398-5348. https://doi.org/10.1108/ILS-04-2020-0100

* Amador, R. (2012). 40 años del Sistema Universidad Abierta de la unam. Crónica histórica. Perfiles Educativos, 34(137), 194-212. https://doi.org/10.22201/ iisue.24486167e.2012.137.34124

* Alaasutari, H. (2020). Abordando la desigualdad en la educación durante y después del covid-19. Los retos de la educación inclusiva. Banco Mundial Blogs. https://cutt.ly/Qn26fEu

* Clark, B. (1997). Las universidades modernas: espacios de investigación y docencia. Miguel Ángel Porrúa; unam.

* Cáceres, M., Lara, L., Iglesias, C. M., García, R., Bravo, G., Cañedo, C. y Valdés Chaviano, O. (2003). La formación pedagógica de los profesores universitarios. Una propuesta en el proceso de profesionalización del docente. Revista Iberoamericana de Educación, 33(1), 1-15. https://doi.org/10.35362/rie3312900

* Camilli-Trujillo, C., Arroyo Resino, D., Asensio Muñoz, I. I, y Mateos Gordo, P. (2020). Hacia la educación basada en la evidencia: Un método y un tema. Revista Electrónica en Educación y Pedagogía, 4(6), 69-85. https://doi.org/10.15658/10.15658/rev. electron.educ.pedagog20.05040606

* Cepal. (2020). La educación en tiempos de la pandemia covid-19. Informe. Santiago, CEPAL-UNESCO. https://cutt.ly/zn9pK5s

* Clade / IIPE-Unesco / OXfam. (2021). Desigualdades educativas en América Latina: tendencias, políticas y desafíos. Campaña Latinoamericana por el Derecho a la Educación / IIPE UNESCO / OXFAM. https://cutt.ly/hnnpHrQ

* Contreras, J. (1994). Enseñanza, currículum y profesorado. AKAL.

* Cobo, C. y Narodowski, M. (2020). El incierto futuro de la educación escolar. Tendencias Pedagógicas, 35, 1-6. https://doi.org/10.15366/tp2020.35.001

* Davies, H., Nutley, S. y Smith, P. (1999) Viewpoint: Editorial: What Works? The Role of Evidence in Public Sector Policy and Practice. Public Money \& Management, 19(1), 3-5. https://doi.org/10.1111/1467-9302.00144

* Díaz-Barriga, A. (2020). La escuela ausente, la necesidad de replantear su significado. En H. Casanova Cardiel (Coord.), Educación y pandemia. Una visión académica (pp. 19-29). UNAM. http://www.iisue.unam.mx/nosotros/covid/ educacion-y-pandemia 
* Díaz-Barriga, A. (2005). El docente y los programas escolares. Lo institucional y lo didáctico. Ediciones Pomares.

- Etikan, I., Abubakar Musa, S. y Sanusi Alkassim, R. (2016). Comparison of Convenience Sampling and Purposive Sampling. American Journal of Theoretical and Applied Statistics, 5(1), 1-4. http://www.doi.org/10.11648/j.ajtas.20160501.11

* Gaceta unam. (2020, 11 junio). Acuerdo por el que se crea la Coordinación de Universidad Abierta, Innovación Educativa y Educación a Distancia. UnAm. https:// cutt.ly/zn9aunU

* Grijalva-Verdugo, A. y Lara- Rivera, J. (2021). Saberes digitales de profesores en formación. Evaluación en una escuela mexicana. Revista Electrónica en Educación y Pedagogía, 5(8), 116-129. https://doi.org/10.15658/rev.electron.educ. pedagog21.04050808

* Gopal, R. Singh, V. y Aggarwal, A. (2021). Impact of online classes on the satisfaction and performance of students during the pandemic period of covid 19. Education and Information Technologies. https://doi.org/10.1007/s10639-021-10523-1

* Flores, P. (2013). El enfoque de la política basado en la evidencia: Análisis de su utilidad para la educación de México. RMIE, 18(56), 265-290. http://www.scielo.org. mx/pdf/rmie/v18n56/v18n56a12.pdf

* Hoodges, C., Moore, S., Lockee, B., Trust, T. y Bond, A. (2020). The Difference Between Emergency Remote Teaching and Online Learning. Educause Review. https://cutt.ly/zn9aDgD

* Kress, G.y Bezemer, J. (2009). Escribir en un mundo de representación multimodal. En J. Kalman y B. Street (Comps.), v. Lectura, escritura y matemáticas como prácticas sociales (pp. 64-83). Siglo xxI.

- Lindblom, C. (1958). Policy Analysis. The American Economic Review, 48(3), 298-312. http://www.jstor.org/stable/1809767

* Loreto, M. (2021). Digitalización acelerada: lo que la pandemia le enseñó a la educación. Blog BID Educación. https://cutt.ly/pn9aNLP

* Luke, B. y Kohnke, L. (2021). Thriving or Surviving Emergency Remote Teaching Necessitated by covid-19: University Teachers' Perspectives. Asia-Pacifico Edu Res, 30(3), 279-287. https://doi.org/10.1007/s40299-021-00567-9

* Mae-Toquero, C. (2021). Experimento de educación remota de emergencia en medio de la pandemia de Covid-19 en instituciones de aprendizaje en Filipinas. International Journal of Educational Research and Innovation (IJERI), 15, 162-176. https://doi.org/10.46661/ijeri.5113

* Moore, S. (2021) The future of pandemics. News medical. https://cutt.ly/on9a7mj

* oECD. (2021). The state of school education: One year into the covid pandemic. Preliminary results, March 2021. oECD. https://cutt.ly/fn9seJc

* Pardo, H. y Cobo, C. (2020). Expandir la universidad más allá de la enseñanza remota de emergencia Ideas hacia un modelo híbrido post-pandemia. Outliers school. https://cutt.ly/cn9soyT 
* PNUD. (2020). Informe sobre desarrollo humano. La próxima frontera. El desarrollo humano y el antropoceno. http://hdr.undp.org/sites/default/files/hdr_2020_overview_spanish.pdf

- Pokhrel, S. y Chhetri, R. (2021). A Literature Review on Impact of covid-19 Pandemic on Teaching and Learning. Higher Education for the Future, 8(1), 133-141. https://doi. org/10.1177/2347631120983481

* Reich, J. (2020). Failure to Disrupt: Why Technology Alone Can't Transform Education. Harvard University Press.

- Sánchez Mendiola, M., Martínez Hernández, A., Torres Carrasco, R., De Agüero Servín, M., Hernández Romo, A., Benavides Lara, M., Rendón Cazales, V. y Jaimes Vergara, C. (2020). Retos educativos durante la pandemia de coviD-19: una encuesta a profesores de la unam. Revista Digital Universitaria, 21(3), 1-24, http:// doi.org/10.22201/codeic.16076079e.2020.v21n3.a12

* Saavedra, J. (2020). Una crisis educativa silenciosa y desigual. Y las semillas para su solución. Blogs Banco Mundial. https://cutt.ly/In9sxph

* Sabatier, P. (1991). Toward Better Theories of the Policy Process. Political Science and Politics, 24(2), 147-156. https://doi.org/10.2307/419923

- Sieber, J. (2005). Misconceptions and Realities about Teaching Online. Science and Engineering Ethics, 11, 329-340 https://doi.org/10.1007/s11948-005-0002-7

* Silas, J. y Vázquez, S. (2020). El docente universitario frente a las tensiones que le plantea la pandemia. Resultados de un estudio mexicano/latinoamericano. RLEE, 50(número especial), 89-120. https://doi.org/10.48102/rlee.2020.50.ESPECIAL.97

- Tesar, M. (2020). Towards a Post-Covid-19 'New Normality?': Physical and Social Distancing, the Move to Online and Higher Education. Policy Futures in Education, 18(5), 556-559. https://doi.org/10.1177/1478210320935671

- Trust, R. y Whaleen, J. (2020). Should Teachers be Trained in Emergency Remote Teaching? Lessons Learned from the CovID-19 Pandemic. Journal of Technology and Teacher Education, 28(2), 189-199 https://www.learntechlib.org/primary/p/215995/

* Velázquez-Cigarroa, E. y Tello-García, E. (2021). Prospección del sistema educativo mexicano a partir de las experiencias aprendidas por el covid-19. Revista Electrónica en Educación y Pedagogía, 5(8), 145-157. https://doi.org/10.15658/rev.electron. educ.pedagog21.04050810

* Whittle, C. Tiwari, S. Yan, S. y Williams, J. (2020). Emergency remote teaching environment: a conceptual framework for responsive online teaching in crises. Information and Learning Sciences, 121(5/6), 311-319. https://doi.org/10.1108//LS-04-2020-0099

\section{Cómo CITAR ESTE ARTículo}

* de Agüero Servín, Mercedes, Benavides Lara, Mario Alberto, Rendón Cazales, Jesús, Pompa Mansilla, Maura, Hernández-Romo, Alan Kristian, Hernández-Martínez, Ana María del Pilar y Sánchez-Mendiola, Melchor. (2021, septiembre-octubre). Los retos educativos durante la pandemia de covID-19: segunda encuesta a profesoras y profesores de la UNAM. Revista Digital Universitaria (RDU), 22(5). http://doi. org/10.22201/cuaieed.16076079e.2021.22.5.13 